\title{
Correction to: Minimally invasive treatment of diaphragmatic endometriosis: a 15-year single referral center's experience on 215 patients
}

\author{
Marcello Ceccaroni ${ }^{1} \cdot$ Giovanni Roviglione ${ }^{1}$ (D) Antonino Farulla ${ }^{2} \cdot$ Pietro Bertoglio $^{3} \cdot$ Roberto Clarizia $^{1}$. \\ Andrea Viti $^{3} \cdot$ Daniele Mautone $^{1} \cdot$ Matteo Ceccarello $^{1} \cdot$ Anna Stepniewska $^{1} \cdot$ Alberto Claudio Terzi $^{3}$
}

Published online: 13 January 2021

(c) Springer Science+Business Media, LLC, part of Springer Nature 2021

\section{Correction to: Surgical Endoscopy https://doi.org/10.1007/s00464-020-08186-z}

This article was updated to correct the author listing, where given names and surnames were reversed.

They are correct as displayed here.

Publisher's Note Springer Nature remains neutral with regard to jurisdictional claims in published maps and institutional affiliations.

The original article can be found online at https://doi.org/10.1007/ s00464-020-08186-z.

Giovanni Roviglione

gruvy79@gmail.com

1 Department of Obstetrics and Gynecology, Gynecologic Oncology and Minimally-Invasive Pelvic Surgery, International School of Surgical Anatomy, IRCCS Sacro Cuore Don Calabria Hospital, Via Don Angelo Sempreboni 5, 37024 Negrar di Valpolicella, Verona, Italy

2 Minimally Invasive and Robotic Gynecologic Surgery Unit, Policlinico di Modena University of Modena and Reggio Emilia, Modena, Italy

3 Division of Thoracic Surgery, IRCCS Sacro Cuore Don Calabria Hospital, Negrar di Valpolicella, Verona, Italy 